\title{
Proteome profiling of low grade serous ovarian cancer
}

\author{
Haniyeh Bashi zadeh fakhar ${ }^{1}$, Hakimeh Zali ${ }^{2}$, Mostafa Rezaie-Tavirani ${ }^{1 *}$, Roya Faraji Darkhaneh ${ }^{3}$ and \\ Babak Babaabasi ${ }^{4}$
}

\begin{abstract}
Background: Serous carcinoma, the subtype of ovarian cancer has the highest occurrence and mortality in women. Proteomic profiling using mass spectrometry (MS) has been used to detect biomarkers in tissue s obtained from patients with ovarian cancer.

Thus, this study aimed at analyzing the interactome (protein-protein interaction (PPI)) and (MS) data to inspect PPI networks in patients with Low grade serous ovarian cancer.

Methods: For proteome profiling in Low grade serous ovarian cancer, 2DE and mass spectrometry were used. Differentially expressed proteins which had been determined in Low grade serous ovarian cancer and experimental group separately were integrated with PPI data to construct the (QQPPI) networks.

Results: Six Hub-bottlenecks proteins with significant centrality values, based on centrality parameters of the network (Degree and between), were found including Transgelin (TAGLN), Keratin (KRT14), Single peptide match to actin, cytoplasmic 1(ACTB), apolipoprotein A-I (APOA1), Peroxiredoxin-2 (PRDX2), and Haptoglobin (HP).

Discussion: This study showed these six proteins were introduced as hub-bottleneck protein. It can be concluded that regulation of gene expression can have a critical role in the pathology of Low-grade serous ovarian cancer.
\end{abstract}

Keywords: Proteome, Profiling, Low grade serous ovarian cancer

\section{Introduction}

Ovarian cancer is the fifth cause of death among other cancers in American women [1]. In Iran, it is the eighth common cancer [2].

Epithelial cell tumors are the most common type of ovarian cancer, which are responsible for $90 \%$ of women's ovarian and 1/4 genital malignancies. Epithelial ovarian cancer had various types including serous (the most common, $50 \%$ of all ovarian cancers), mucinous (15-20\%), endometriosis (25-10\%), clear cell (10.5\%), undifferentiated (5\%) and Brenner (5\%). Serous cancer is usually moderate and diagnosed at lower ages and stage with a better prognosis [3].

Furthermore, serous carcinoma has the highest occurrence and mortality comprising $90 \%$ of all deaths due to

\footnotetext{
* Correspondence: m.Tavirany@gmail.com

${ }^{1}$ Proteomics Research Center, Shahid Beheshti University of Medical Sciences, Tehran, Iran

Full list of author information is available at the end of the article
}

ovarian cancer. However, its origin and rapid progression are poorly understood $[4,5]$.

Frequently the lack of reliable clinical tests and the latent stages of the disease worsen most cases of ovarian cancer (68\%), more than $95 \%$ of serous who are diagnosed with poor survival chance and metastatic condition [6-8]. According to reports, women diagnosed with low grade ovarian cancer have a 5-year survival rate of approximately $80-90 \%$, but this decreases significantly to $20-30 \%$ in late-stage diagnoses [7-9]. Despite of all advances in screenings and available therapies, none of the existing screening methods facilitate prompt diagnosis and confirmation of the ovarian cancer [10] while apparently early diagnosis is a critical factor in reducing the mortalities due to ovarian cancer [11].

Based on Staging defined by the FIGO (International Federation of Gynecology and Obstetrics) system, lowgrade disease including (stage I and II) describes a tumor that is localized in its original site, with no spread to lymph nodes or other body areas. The low-grade disease 
has the chance of a cure if the malignancy can be surgically removed successfully [12].

Many researchers have considered using CA-125 as a biomarker for early diagnosis [13, 14]. However, CA-125 is most of the times falsely negative infertile women with serous ovarian cancer and in Low grade serous ovarian cancer and CA-125 is positive in benign diseases. Thus, it is not sensitive enough for usage in general screening $[15,16]$.

Recent technologies have made performing complicated studies easier in order to specify the subtypes of serous ovarian cancer using genomic, transcriptomic and proteomic [17]. Specifically, proteomic profiling of serous ovarian cancer has mainly revolved around the analysis of serous ovarian cancer cell lines, tissues, and proximal fluids, urines and cyst fluid by using mass spectrometry (MS) [18-20]. Recently, the so-called, mass-spectrometry-based quantitative proteomics is the common strategy in identifying the proteins and their alterations [21, 22].

This study reports on proteomics profiling study of Low grade serous ovarian cancer by using integrate interactome (protein-protein interaction (PPI)) and (MS) data to construct and analyze PPI networks for Low grade serous ovarian cancer from controls with $100 \%$ accuracy, sensitivity, and specificity possible through panel markers.

As all previous studies have considered metastasis or high stage ovarian cancer, this study has dedicated its focus on low grade ovarian serous.

\section{Materials}

All chemicals used in this study were purchased from Sigma-Aldrich (St. Louis, MO, USA) with exceptions noted. Criterion precast polyacrylamide gels, TGS and XT MES electrophoresis running buffers, Ready Strip ${ }^{\text {Tx }}$ IPG strips, mineral oil, dithiothreitol (DTT), iodoacetamide (IA), Biolytic, and urea were purchased from BioRAD.

\section{Sampling}

After obtaining informed consent form, 10 healthy volunteers (women without low-grade serous ovarian cancer) entered in the study for ovarian tissue surgery; a sample size of $1 \times 1 \mathrm{~cm}$ was taken. A part of it was transferred to the pathology lab for natural tissue confirmation. The other part was immediately transferred to the liquid nitrogen reservoir to be transmitted to the proteomics lab at the Shahid Beheshti University Clinical Projective Research Center, Tehran. Tumor sampling was conducted on 10 patients during surgery. A part of the tissue was sent to a pathology lab to be examined pathologically (confirmation of cancer). The other sample was then transferred to a liquid nitrogen tank at $96^{\circ} \mathrm{C}$ for less than $2 \mathrm{~min}$, and a proteomic test was sent to the proteomics lab at the Shahid Beheshti University of Medical Sciences, Tehran.

\section{Experimental group}

At first, all specimens were examined for the level and type of cancer by an independent pathologist and then ten women with Low grade serous ovarian cancer (and 10 without) were selected for this study. We didn't used biochemical criteria.

They were referred to the hospitals of Guilan University of Medical Sciences in Rasht from 2014 to 2015 were sampled. Examples of scientific information relevant to the study variables, without restriction of any kind, patient and personal information were used only by a specific code and were normally archived.

\section{Preparation}

For protein extraction frozen healthy and cancerous tissues of patients under liquid nitrogen, the condition was powdered completely. The resulting powder with lubricating buffer containing Tris- $\mathrm{HCl}$, magnesium chloride, EDTA and phenyl methyl sulfonyl fluoride (PMSF) and $5 \mathrm{~mm}$ beta-mercaptoethanol, $0.5 \%$ CHAPS, and 10\% glycerol was kept in ice for $30 \mathrm{~min}$. Then, the solution was centrifuged in 16,000 Ground at $4{ }^{\circ} \mathrm{C}$ for 30 minutes, and protein assay was performed by Bradford technique [23]. The sample was also taken during the dewatering. After quantification of proteins, the supernatants were kept at $-20{ }^{\circ} \mathrm{C}$ until used for electrophoresis.

\section{Two-dimensional gel electrophoresis}

In each group, $400 \mu \mathrm{g}$ of the extracted protein was separately mixed with rehydration buffer and The $\mathrm{pH}$ is applied to 3 to $10 \mathrm{~cm}$ (IPG) strip and was passively rehydrated with above sample solution overnight at room temperature. Isoelectrofocusing (IEF) was performed by increasing the voltage From 500 to $8000 \mathrm{~V}$ during the first $3 \mathrm{~h}$, and then a gradient pattern was used to achieve $8000 \mathrm{~V}$ for $3 \mathrm{~h}$. Following the IEF, IPG strips were incubated equilibration buffer containing $6 \mathrm{M}$ urea, $30 \%$ glycerol, 2\% SDS, $2 \%$ DTT and then alkylated for $20 \mathrm{~min}$ in the same buffer with $2.5 \%$ iodoacetamide instead of DTT, to separate the second dimension; the treated strips were transferred onto 12\% SDSPolyacrylamide slab gel and sealed with $1 \%$ agarose. The gels run in $2.5 \mathrm{~W}$ each gel for $30 \mathrm{~min}$ and $15 \mathrm{~W}$ each gel as far as the blue front of thebromophenol reaches the end of the gel. The analytical gels were stained with Coomassie blue. Gels were scanned using Bio-Rad Image Scanner and Spot detection, matching, and quantitative gel analyses were carried out with Nonlinear Progenesis software. 


\section{Protein identification by MALDI-TOF/TOF}

In-gel protein digestion was performed according to Zhou et al. with minor modifications [24]. The data search was conducted on GPS Explorer (Version 3.6, AB SCIEX). Using the search engine Mascot (Version 2.2, Matrix Science, London, UK) and the International Protein Index (IPI) database (vision 3.64, 39,871 sequences, http://www. ebi.ac.uk/IPI) identify the peptides and protein identifications. The identification of the general protein was based on two or more peptides, whose ionic scores were higher than the statistical threshold. $(p<0.05)$.

\section{Statistical analysis}

Scanned 2DE gels were analyzed by using Non-linear Prognosis Same Spot software to compare gels together and compare the spots in one statement in gels and get the density of the same spot in each of gel. To detect significant differences between the experimental groups, analysis of variance (ANOVAs) were used. A $p$-value < 0.05 was considered to be statistically significant. Statistics were presented as means $\pm \mathrm{SE}$. Other multivariate analyses on protein expressions use hierarchical clustering and principal components analysis.

\section{Bioinformatics}

Identified proteins were used to determine predicted interactions with other proteins. This functional protein association network for each entry was obtained by searching "the string" online database (http://string-db. org). The sub-networks of QQPPI were constructed and visualized by Cytoscape software [25].

The following parameters were calculated to determine biologically significant nodes. Hub and bottleneck nodes were extracted from the networks in two steps; (first) In the networks, nodes with degree greater than or equal to the sum of mean and twice the standard deviation (S.D.), i.e., mean C $2 *$ S.D. of the degree distribution, were considered as hubs [26]. (Second) We defined bottlenecks as the proteins that were in the top 5\% in terms of betweenness centrality. After all the identified proteins were matched to specific processes or functions by searching the $\mathrm{GO}$ in CluGO/Clupedia.

\section{Result}

After ovarian tissue extraction, proteome profile of low-grade serous ovarian cancer was determined and analyzed by using Prognosis Same Spots software. The results showed that spots had a statistically significant variation with relative abundance $(p<0.05)$. As is shown in the Fig. 1, among 212 proteins 41 differentially changed expression protein $(\mathrm{FC}>2)$ were identified. Among 41 spots 18 top changed expression spots were investigated by MS, and the final determined proteins are tabulated in Table 1 . The 10 significant differentially expressed proteins were imported in the string, and the constructed network including 1138 nodes and 1449 edges (the network is not shown) was analyzed. The hubs, bottlenecks, and hub-bottlenecks were represented in the Tables 2, 3 and 4. For more resolution, the hub and bottleneck nodes were included in a subnetwork (see Fig. 2). The finding indicates that 6 Hubbottlenecks proteins including Transgelin (TAGLN), Keratin (KRT14), Single peptide match to actin cytoplasmic 1(ACTB), apolipoprotein A-I (APOA1), Peroxiredoxin-2 (PRDX2), and Haptoglobin (HP) (see Table 5) are query proteins which were identified by MS analysis.

Gene ontology analysis counting biological processes, molecular function, and cellular component via Clupe$\mathrm{dia} / \mathrm{CluGO}$ were applied for the six common proteins in the central nodes and the significant differentially expressed proteins (see Figs. 3, 4 and 5).

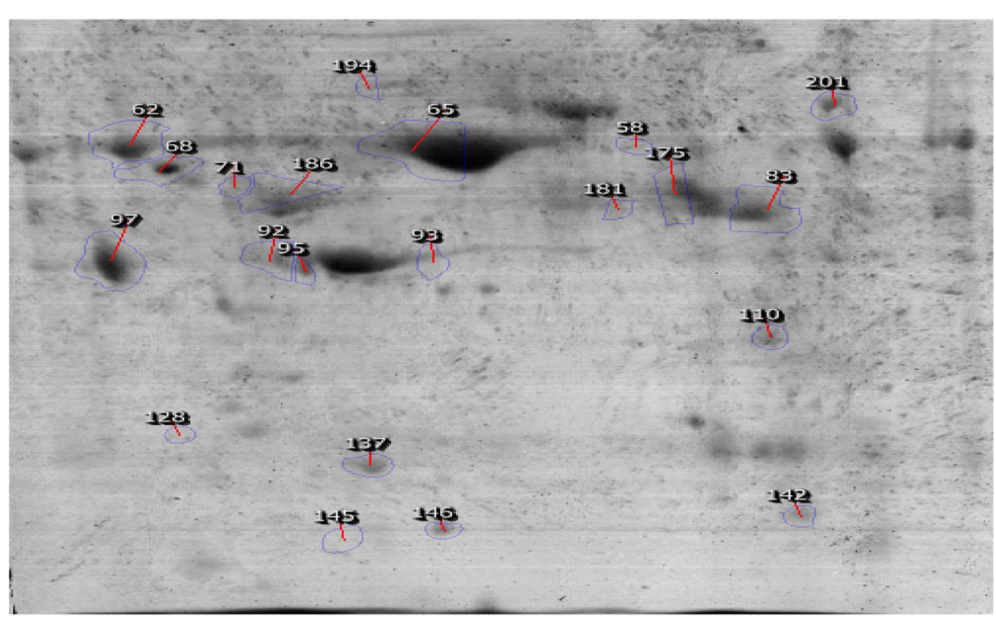

Fig. 118 Protein submitted for MS-identified 
Table 1 Proteins identified by mass spectrometry in two groups

\begin{tabular}{|c|c|c|c|c|c|}
\hline Protein Num & Kind of Protein & Protein expression & Fold & Size & $\begin{array}{l}\text { Weight } \\
\text { Dalton }\end{array}$ \\
\hline $62-64,181$ & albumin & Decrease & $2-2.1-1.1$ & 609 & 69,367 \\
\hline 201 & albumin & Increase & 1.8 & 609 & 69,367 \\
\hline 83 & Ig gamma-1 chain $C$ region & Increase & 1.5 & 334 & 36,500 \\
\hline 92 & Haptoglobin & Decrease & 1.3 & 406 & 45,205 \\
\hline 95 & Haptoglobin & Increase & 1.4 & 406 & 45,205 \\
\hline 93 & Single peptide match to actin, cytoplasmic 1 & Decrease & 1.7 & 402 & 41,785 \\
\hline 110 & Glyceraldehyde-3-phosphate dehydrogenase & Increase & 1.8 & 335 & 36,053 \\
\hline 137 & Apolipoprotein A-I & Increase & 1.6 & 267 & 30,778 \\
\hline 142 & Transgelin & Increase & 1.0 & 201 & 22,611 \\
\hline 146 & Peroxiredoxin-2 & Increase & 1.8 & 198 & 21,892 \\
\hline 175 & Keratin & Decrease & 1.5 & 472 & 51,561 \\
\hline 186 & Alpha-1-antitrypsin & Increase & 1.4 & 503 & 54,030 \\
\hline
\end{tabular}

\section{Discussion}

Pathogenesis of serous ovarian cancer was studied by the proteomic approach to identify the alteration in gene expression between normal and low-grade cancer tissues [27]. So far, not many approaches have been devised to identify the specific differential protein expression between primary and recurrent serous in tissues of these patients [28]. For example, using quantitative proteomics via ICAT, Pan et al. compared the expression between a chemosensitive and a chemoresistant tissue [29]. Another study used paired primary and recurrent postchemotherapy samples from high-grade serous ovarian cancer patients to identify numerous proteins elevated in recurrent tissues [30].

In our study, we found 18 proteins with different expression compared to normal and low grade of cancer tissue, which is represented in Fig. 1. There is just one study, which reported 18 metabolites differentiated in Low gradetumors in comparison to control mice, which were performed by Jones $\mathrm{Cm}$ et al. [11].

We constructed PPI networks of abnormally expressed proteins in the paired low garde tumor by integrating interactome and mass spectrometry data. Based on proteins which had identified by MALDI-TOF and QQPPI networks analysis, we found 6 Hub-bottlenecks proteins with significant centrality values, based on centrality parameters of the network (degree and betweenness), such as Transgelin (TAGLN), Keratin (KRT14), Single peptide match to actin, cytoplasmic 1 (ACTB), apolipoprotein AI (APOA1), Peroxiredoxin-2 (PRDX2), and Haptoglobin (HP) (Table 5).

All detected proteins in our study play an important role throughout the network because of that they all act as hubs. Transgelin is the protein according to Table 5 as the important protein in the network and overexpressed in cancer cells. Mohamed El Ayed has found
Transgelin in ovarian cancer proteome [31], Zhou et al. have studied the mechanism of Transgelin in colorectal cancer, which has low endogenous levels, led to increased invasiveness, growth at low density [32]. In other human cancers such as lung adenocarcinoma, the expression of transgelin- 2 has been reported. This protein has been proposed to be related to the increase in migratory and invasive abilities [33] and repressing genes involved in tumor progression [34]. In addition, several investigations have shown that Transgelin in normal and cancer cells directly interact with the actin, and alter the motility of the cells [35-38].

Keratin, has been identifies as the most commonly used marker to identify tumor cells from carcinomas and as standard detection marker for disseminated tumor cells and circulating tumor cells [39]. Cytoskeleton has a vital role in disseminated tumor cells and circulating tumor cells [40]. Keratin is one of the candidate proteins that signal the cytoskeleton [41, 42]. El Ayed reported keratin's increasing expression in ovarian cancer [31], such as Keshamouni through analysis of human lung adenocarcinoma cell line [33]. In another study, Joosse and et al. after reviewing keratin expression during metastatic progression of breast cancer found primary breast carcinomas changes in keratin expression during metastatic progression to the lymph nodes [43].

Cytoplasmic 1 is the next protein that previously was reported by Toyama's study on ovarian cancers [44, 45]. One study on 13 prostate cancer specimens reported cytoplasmic expression [46]. In another study on human lung adenocarcinoma cell line, this differentiation was accompanied by the modification in the expression of several cytoskeleton proteins such as cytoplasmic [33].

We identified Apolipoprotein A-I increasing expressed proteins in Low grade serous ovarian cancer such as Kristjansdottir's study [21]. In another study about protein 
Table 2 Hub proteins with significant centrality values, based on degree

\begin{tabular}{|c|c|c|c|}
\hline Num & Hub genes & Protein name & Degree \\
\hline 1 & ACTB & actin beta(ACTB) & 913 \\
\hline 2 & APOA1 & apolipoprotein A1(APOA1) & 261 \\
\hline 3 & PRDX2 & peroxiredoxin 2(PRDX2) & 148 \\
\hline 4 & TAGLN & transgelin(TAGLN) & 70 \\
\hline 5 & $\mathrm{HP}$ & haptoglobin(HP) & 58 \\
\hline 6 & KRT1 & keratin 1(KRT1) & 31 \\
\hline 7 & ACTG1 & actin gamma 1 (ACTG1) & 21 \\
\hline 8 & ERBB2 & erb-b2 receptor tyrosine kinase 2(ERBB2) & 16 \\
\hline 9 & CFL2 & cofilin 2(CFL2) & 14 \\
\hline 10 & CFL1 & cofilin 1(CFL1) & 13 \\
\hline 11 & LCAT & lecithin-cholesterol acyltransferase(LCAT) & 12 \\
\hline 12 & APP & amyloid beta precursor protein(APP) & 11 \\
\hline 13 & ESR1 & estrogen receptor 1(ESR1) & 11 \\
\hline 14 & APOE & apolipoprotein E(APOE) & 11 \\
\hline 15 & DSTN & destrin, actin depolymerizing factor(DSTN) & 11 \\
\hline 16 & NCF1 & neutrophil cytosolic factor 1(NCF1) & 10 \\
\hline 17 & VCAM1 & vascular cell adhesion molecule 1(VCAM1) & 10 \\
\hline 18 & $\mathrm{FBXO} 25$ & F-box protein 25(FBXO25) & 10 \\
\hline 19 & SMAD3 & SMAD family member 3(SMAD3) & 9 \\
\hline 20 & $\mathrm{ABCA} 1$ & ATP binding cassette subfamily A member 1 (ABCA1) & 9 \\
\hline 21 & SMARCA4 & SWI/SNF related, matrix associated, actin dependent regulator of chromatin, subfamily a, member 4(SMARCA4) & 9 \\
\hline 22 & TINF2 & TERF1 interacting nuclear factor 2(TINF2) & 8 \\
\hline 23 & FN1 & fibronectin 1(FN1) & 7 \\
\hline 24 & YWHAZ & tyrosine 3-monooxygenase/tryptophan 5-monooxygenase activation protein zeta(YWHAZ) & 7 \\
\hline 25 & NOS3 & nitric oxide synthase 3(NOS3) & 7 \\
\hline 26 & $T T R$ & transthyretin(TTR) & 6 \\
\hline 27 & JUN & Jun proto-oncogene, AP-1 transcription factor subunit(JUN) & 6 \\
\hline 28 & IQGAP1 & IQ motif containing GTPase activating protein 1(IQGAP1) & 6 \\
\hline 29 & PFN1 & profilin 1(PFN1) & 6 \\
\hline 30 & HNRNPU & heterogeneous nuclear ribonucleoprotein U(HNRNPU) & 6 \\
\hline 31 & POLR2A & RNA polymerase II subunit A(POLR2A) & 6 \\
\hline 32 & PPARG & peroxisome proliferator activated receptor gamma(PPARG) & 5 \\
\hline 33 & SCARB1 & scavenger receptor class B member 1(SCARB1) & 5 \\
\hline 34 & CMTM5 & CKLF like MARVEL transmembrane domain containing 5(CMTM5) & 5 \\
\hline 35 & HSPA8 & heat shock protein family A (Hsp70) member 8(HSPA8) & 5 \\
\hline 36 & NSMAF & neutral sphingomyelinase activation associated factor(NSMAF) & 5 \\
\hline 37 & TRAF3IP1 & TRAF3 interacting protein 1(TRAF3IP1) & 5 \\
\hline 38 & SS18 & SS18, nBAF chromatin remodeling complex subunit(SS18) & 5 \\
\hline 39 & SYT-SSX1 & Synovial sarcoma typically & 5 \\
\hline 40 & CAP2 & CAP, adenylate cyclase-associated protein, 2 (yeast)(CAP2) & 5 \\
\hline 41 & MYH9 & myosin heavy chain $9(\mathrm{MYH} 9)$ & 5 \\
\hline 42 & EMD & emerin(EMD) & 5 \\
\hline 43 & FBLN1 & fibulin 1(FBLN1) & 4 \\
\hline 44 & KRT9 & keratin 9(KRT9) & 4 \\
\hline
\end{tabular}


Table 2 Hub proteins with significant centrality values, based on degree (Continued)

\begin{tabular}{llll}
\hline Num & Hub genes & Protein name & Degree \\
\hline 45 & ACD & adrenocortical dysplasia homolog(ACD) & 4 \\
46 & POT1 & protection of telomeres 1(POT1) & 4 \\
47 & RAD52 & RAD52 homolog, DNA repair protein(RAD52) & 4 \\
48 & CDC37 & cell division cycle 37(CDC37) & 4 \\
49 & NFKB1 & nuclear factor kappa B subunit 1(NFKB1) \\
50 & OTUB1 & OTU deubiquitinase, ubiquitin aldehyde binding 1(OTUB1) & 4 \\
51 & UTY & ubiquitously transcribed tetratricopeptide repeat containing, Y-linked(UTY) & 4 \\
52 & GRB2 & growth factor receptor bound protein 2(GRB2) \\
53 & MIS12 & MIS12, kinetochore complex component(MIS12) & 4 \\
54 & TXN & thioredoxin(TXN) & 4 \\
55 & APOC1 & apolipoprotein C1(APOC1) \\
56 & FGA & fibrinogen alpha chain(FGA) \\
57 & KRT16 & keratin 16(KRT16) & 4 \\
58 & APOB & apolipoprotein B(APOB) & 4 \\
59 & NAXE & NAD(P)HX epimerase(NAXE) & 4 \\
\hline
\end{tabular}

expression patterns associated with advanced stage ovarian cancer by Cortes, Apolipoprotein A-I was identified by their proteomic screening, which had increased expression in ovarian cancer samples $[47,48]$, and they suggested that protein suitable for further investigation [49]. Similarly, apolipoprotein A1 has been detected in conjunction with transthyretin and transferrin in low grade mucinous tumors [50]. ApoA-I candiminish the expression of surface molecules such as CD1a, CD80, CD86, and HLA-DR in dendritic cells, and it stimulates the production of IL -10 [51].

Furthermore, we had an increase in the expression of Ig gamma-1 chain $C$ region in Low grade serousovarian cancer such as Cortesi study [48].

We reported peroxiredoxin-2 increasing expressions such as Kristjansdottir [21], Cortesi study [48] and Atsuhiko et al. on subtypes of ovarian carcinoma [20]. Moreover, high levels of antioxidative enzymes, such as glutathione peroxidase3, peroxiredoxin-2, peroxiredoxin6 , and superoxide dismutase, It may be responsible for resistance to apoptosis caused by oxidative stress or chemotherapy $[52,53]$.

One protein identified in our study was haptoglobin-1 similar to Ahmed et al. on serum of ovarian cancer patients [54]. Cortesi identified two spots by a single peptide as haptoglobin-related protein [48]. The haptoglobin is one of the richest glycoproteins secreted by the liver [55], it is reasonable to hypothesize that enhanced hepatic synthesis of the protein will occur due to an acute phase response in ovarian cancer patients resulting in elevated serum haptoglobin precursor concentration [56]. On the other hand, Haptoglobin level was shown to be affected by the amount of tumor burden and was not dependent on the histologic type or grade of ovarian malignancy [57].

On the other hand, six of the above-mentioned proteins have been identified at high grade and metastatic of serous ovarian cancer. Biton and et al. in study on high grade Bladder Tumor Transcriptome and serous ovarian cancer, characterized the luminal and basal-like subtypes of muscle-invasive bladder cancers according to the components (such as transgelin) which showed luminal tumors had lost morphological differentiation [58]. Several studies have investigated the expression of keratin in high grade ovarian cancer $[59,60]$. Capochichi and etal in study on Overexpression and cytoplasmic localization of caspase- 6 showed that it is associated with lamin A degradation in set of high grade ovarian cancers showed that it may be involved in lamin A degradation and deficiency observed in some ovarian cancer cells [61].

Many studies have reported that Apolipoprotein A-I (ApoAI) levels have been increased in early grade ovarian serous carcinoma patients [62]. Braiacu showed that several lipids (such as Apolipoprotein A) had progressive alterations in high-grade ovarian serous cancer patients with more advanced disease and poorer overall survival [63]. Elevated expression of PRDX3, PRDX5, and PRDX6 mRNAs showed poorer overall survival (OS); PRDX5 and PRDX6 also predicted poor progression-free survival (PFS) for ovarian cancer patients. Furthermore, PRDX3 played significant prognostic roles, particularly in poor differentiation and late-stage serous ovarian cancer patients [64].

In a study by Mahyuddin and etal, they demonstrated the presence of haptoglobin in ovarian cyst fluid of 
Table 3 Bottlenecks proteins with significant centrality values, based on betweeness

\begin{tabular}{|c|c|c|c|}
\hline Num & Bottleneck genes & Protein names & Betweeness \\
\hline 1 & ACTB & actin beta(ACTB) & 0.873 \\
\hline 2 & APOA1 & apolipoprotein A1(APOA1) & 0.222 \\
\hline 3 & PRDX2 & peroxiredoxin 2(PRDX2) & 0.202 \\
\hline 4 & TAGLN & transgelin(TAGLN) & 0.114 \\
\hline 5 & $\mathrm{HP}$ & haptoglobin(HP) & 0.048 \\
\hline 6 & KRT1 & keratin 1(KRT1) & 0.036 \\
\hline 7 & FN1 & fibronectin 1(FN1) & 0.026 \\
\hline 8 & APP & amyloid beta precursor protein(APP) & 0.026 \\
\hline 9 & ESR1 & estrogen receptor 1(ESR1) & 0.026 \\
\hline 10 & UCHL5 & ubiquitin C-terminal hydrolase L5(UCHL5) & 0.026 \\
\hline 11 & FBLN1 & fibulin 1(FBLN1) & 0.017 \\
\hline 12 & TTR & transthyretin(TTR) & 0.016 \\
\hline 13 & KRT9 & keratin 9(KRT9) & 0.016 \\
\hline 14 & VCP & valosin containing protein(VCP) & 0.016 \\
\hline 15 & BAZ1B & bromodomain adjacent to zinc finger domain 1B(BAZ1B) & 0.016 \\
\hline 16 & APOE & apolipoprotein E(APOE) & 0.009 \\
\hline 17 & CFL1 & cofilin 1(CFL1) & 0.008 \\
\hline 18 & NCF1 & neutrophil cytosolic factor 1(NCF1) & 0.008 \\
\hline 19 & TINF2 & TERF1 interacting nuclear factor 2(TINF2) & 0.008 \\
\hline 20 & $A C D$ & adrenocortical dysplasia homolog(ACD) & 0.008 \\
\hline 21 & POT1 & protection of telomeres 1(POT1) & 0.008 \\
\hline 22 & PRKCD & protein kinase $C$ delta(PRKCD) & 0.008 \\
\hline 23 & ISG15 & ISG15 ubiquitin-like modifier(ISG15) & 0.008 \\
\hline 24 & ENO1 & enolase 1(ENO1) & 0.008 \\
\hline 25 & TPM2 & tropomyosin 2 (beta)(TPM2) & 0.008 \\
\hline 26 & LMOD1 & leiomodin 1(LMOD1) & 0.008 \\
\hline 27 & JUN & Jun proto-oncogene, AP-1 transcription factor subunit(JUN) & 0.007 \\
\hline 28 & GPX4 & glutathione peroxidase 4(GPX4) & 0.006 \\
\hline 29 & HINT1 & histidine triad nucleotide binding protein 1(HINT1) & 0.006 \\
\hline 30 & VCAM1 & vascular cell adhesion molecule 1(VCAM1) & 0.005 \\
\hline 31 & MAP1LC3A & microtubule associated protein 1 light chain 3 alpha(MAP1LC3A) & 0.005 \\
\hline 32 & MAP1LC3B & microtubule associated protein 1 light chain 3 beta(MAP1LC3B) & 0.005 \\
\hline 33 & GABARAPL2 & GABA type A receptor associated protein like 2(GABARAPL2) & 0.005 \\
\hline 34 & GABARAPL1 & GABA type A receptor associated protein like 1(GABARAPL1) & 0.005 \\
\hline 35 & GABARAP & GABA type A receptor-associated protein(GABARAP) & 0.005 \\
\hline 36 & PPARG & peroxisome proliferator activated receptor gamma(PPARG) & 0.005 \\
\hline 37 & YWHAZ & tyrosine 3-monooxygenase/tryptophan 5-monooxygenase activation protein zeta(YWHAZ) & 0.005 \\
\hline 38 & RAD52 & RAD52 homolog, DNA repair protein(RAD52) & 0.005 \\
\hline 39 & CDC37 & cell division cycle 37(CDC37) & 0.005 \\
\hline 40 & NFKB1 & nuclear factor kappa B subunit 1(NFKB1) & 0.005 \\
\hline 41 & OTUB1 & OTU deubiquitinase, ubiquitin aldehyde binding 1(OTUB1) & 0.005 \\
\hline 42 & UTY & ubiquitously transcribed tetratricopeptide repeat containing, Y-linked(UTY) & 0.005 \\
\hline 43 & ITGA4 & integrin subunit alpha 4(ITGA4) & 0.005 \\
\hline 44 & COPS5 & COP9 signalosome subunit 5(COPS5) & 0.005 \\
\hline
\end{tabular}


Table 3 Bottlenecks proteins with significant centrality values, based on betweeness (Continued)

\begin{tabular}{llll}
\hline Num & Bottleneck genes & Protein names & Betweeness \\
\hline 45 & HSPA5 & heat shock protein family A (Hsp70) member 5(HSPA5) & 0.005 \\
46 & CUL2 & cullin 2(CUL2) & 0.005 \\
47 & CDK2 & cyclin dependent kinase 2(CDK2) & 0.005 \\
48 & CUL1 & cullin 1(CUL1) & 0.005 \\
49 & CAND1 & cullin associated and neddylation dissociated 1(CAND1) & 0.005 \\
50 & ANXA2 & annexin A2(ANXA2) & 0.005 \\
51 & BCAR1 & BCAR1, Cas family scaffolding protein(BCAR1) & 0.005 \\
52 & PCMT1 & protein-L-isoaspartate (D-aspartate) O-methyltransferase(PCMT1) & 0.004 \\
53 & SMAD3 & SMAD family member 3(SMAD3) & 0.004 \\
55 & GRB2 & growth factor receptor bound protein 2(GRB2) & 0.004 \\
56 & ITGAM & integrin subunit alpha M(ITGAM) & 0.004 \\
\hline
\end{tabular}

Table 4 Proteins with more hub and bottlenecks

\begin{tabular}{|c|c|c|c|c|}
\hline Num & Hub and bottleneck & Protein Name & Degree & Betweeness \\
\hline 1 & ACTB & actin beta(ACTB) & 913 & 0.873 \\
\hline 2 & APOA1 & apolipoprotein A1(APOA1) & 261 & 0.222 \\
\hline 3 & PRDX2 & peroxiredoxin 2(PRDX2) & 148 & 0.202 \\
\hline 4 & TAGLN & transgelin(TAGLN) & 70 & 0.114 \\
\hline 5 & $\mathrm{HP}$ & haptoglobin(HP) & 58 & 0.048 \\
\hline 6 & KRT1 & keratin 1(KRT1) & 31 & 0.036 \\
\hline 7 & CFL1 & cofilin 1(CFL1) & 13 & 0.008 \\
\hline 8 & APP & amyloid beta precursor protein(APP) & 11 & 0.026 \\
\hline 9 & ESR1 & estrogen receptor 1(ESR1) & 11 & 0.026 \\
\hline 10 & APOE & apolipoprotein E(APOE) & 11 & 0.009 \\
\hline 11 & NCF1 & neutrophil cytosolic factor 1(NCF1) & 10 & 0.008 \\
\hline 12 & VCAM1 & vascular cell adhesion molecule 1(VCAM1) & 10 & 0.005 \\
\hline 13 & SMAD3 & SMAD family member 3(SMAD3) & 9 & 0.004 \\
\hline 14 & TINF2 & TERF1 interacting nuclear factor 2(TINF2) & 8 & 0.008 \\
\hline 15 & FN1 & fibronectin 1(FN1) & 7 & 0.026 \\
\hline 16 & YWHAZ & tyrosine 3-monooxygenase/tryptophan 5-monooxygenase activation protein zeta(YWHAZ) & 7 & 0.005 \\
\hline 17 & TTR & transthyretin(TTR) & 6 & 0.016 \\
\hline 18 & JUN & Jun proto-oncogene, AP-1 transcription factor subunit(JUN) & 6 & 0.007 \\
\hline 19 & PPARG & peroxisome proliferator activated receptor gamma(PPARG) & 5 & 0.005 \\
\hline 20 & FBLN1 & fibulin 1(FBLN1) & 4 & 0.017 \\
\hline 21 & KRT9 & keratin 9(KRT9) & 4 & 0.016 \\
\hline 22 & $A C D$ & adrenocortical dysplasia homolog(ACD) & 4 & 0.008 \\
\hline 23 & POT1 & protection of telomeres 1(POT1) & 4 & 0.008 \\
\hline 24 & RAD52 & RAD52 homolog, DNA repair protein(RAD52) & 4 & 0.005 \\
\hline 25 & CDC37 & cell division cycle 37(CDC37) & 4 & 0.005 \\
\hline 26 & NFKB1 & nuclear factor kappa B subunit 1(NFKB1) & 4 & 0.005 \\
\hline 27 & OTUB1 & OTU deubiquitinase, ubiquitin aldehyde binding 1(OTUB1) & 4 & 0.005 \\
\hline 28 & UTY & ubiquitously transcribed tetratricopeptide repeat containing, Y-linked(UTY) & 4 & 0.005 \\
\hline 29 & GRB2 & growth factor receptor bound protein 2(GRB2) & 4 & 0.004 \\
\hline
\end{tabular}




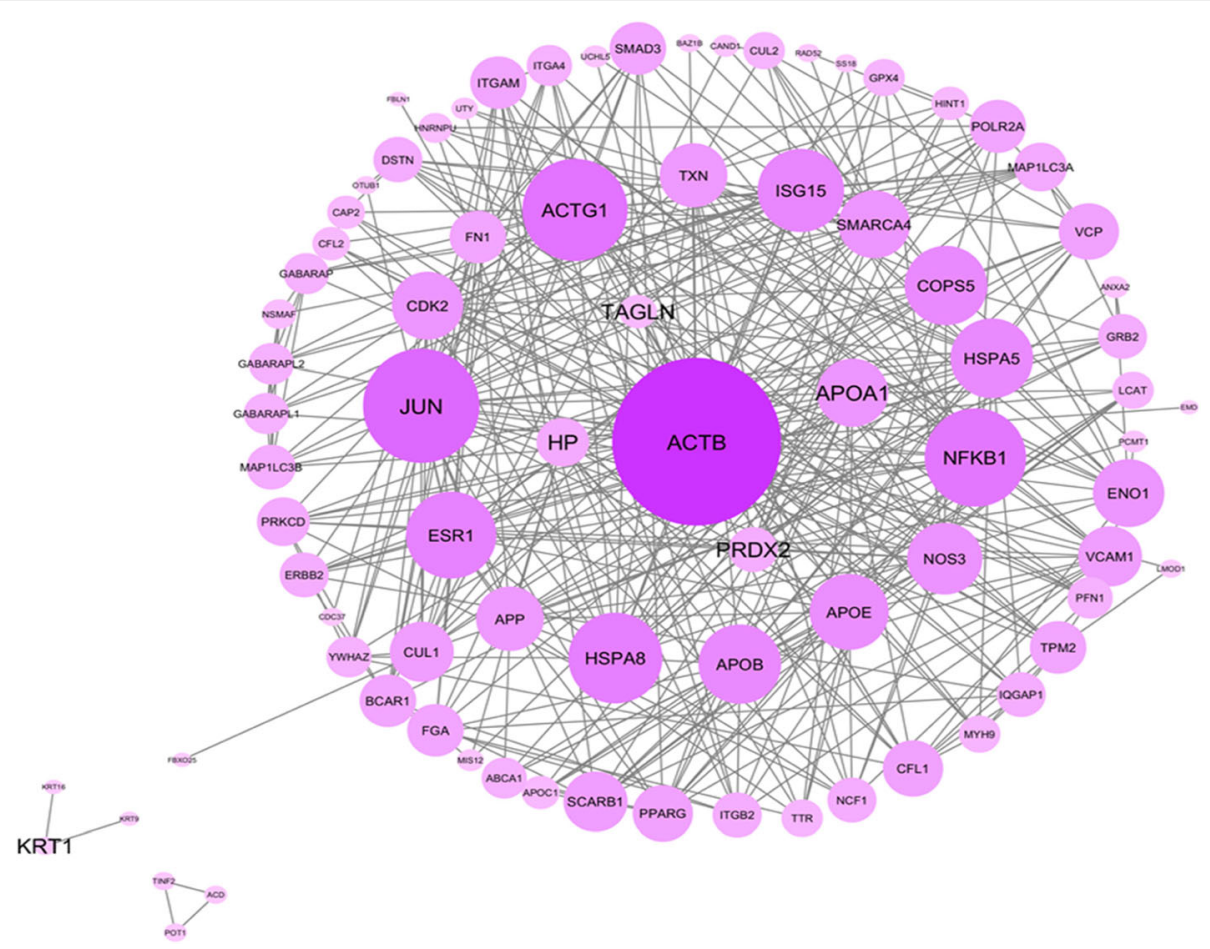

Fig. 2 PPI network for ovarian cancer obtained from MINT, Reactome-FIs and STRING databases by the application of Proteomics Standard Initiative Common Query InterfaCe (PSICQUIC) source for the selected proteins

benign, borderline and malignant epithelial ovarian cancer. The concentration of haptoglobin was significantly raised in ES and LS-EOC compared with benign tumours. They also observed raised haptoglobin concentrations in ovarian cyst fluid of low-volume high-grade ovarian serous cancer [65].

Pathways of hub neighbors were obtained from the QUICK GO (a web-based tool that allows secure browsing of the gene ontology) [66, 67], according to STRING database information (Figs. 3, 4 and 5), related proteins of 6 bottleneck have been predicted.

On the biological process category of gene ontology analysis, we found telomere assembly as a significant factor in low-grade serous ovarian cancer. Telomeres play an important role in controlling the cell proliferation capacity $[68,69]$. According to the Gray's Study

Table 5 Hub-bottlenecks proteins with significant centrality values, based on degree and betweeness

\begin{tabular}{lll}
\hline Protein name & Hub degree & Bottleneck Betweenees \\
\hline TAGLN & 70 & 0.144 \\
KRT14 & 31 & 0.365 \\
ACTB & 913 & 0.873 \\
APOA1 & 261 & 0.222 \\
PRDX2 & 148 & 0.2022 \\
HP & 58 & 0.048 \\
\hline
\end{tabular}

result is on ovarian cancer, Both transcriptional regulation of the human telomerase reverse transcriptase gene and alternative splicing of human telomerase reverse transcriptase transcripts can modulate the assembly of an active enzyme [70].

Cellular response to nitrogen starvation is another pathway that we found like Yoshihiro in ovarian cancer [71]. The mTOR complex 1 (mTORC1) pathway promotes cell growth in response to many cues. GATOR1 has GTPase-activating protein (GAP) activity for RagA and $\mathrm{RagB}$, and its components are mutated in human cancer. In cancer cells with inactivating mutations in GATOR1, mTORC1 is hyperactive and insensitive to nitrogen starvation [72].

A key molecule that is produced due to a change in cancer metabolism reduces Nicotinamide Adenine Dinucleotide (NADH), which functions as a cofactor and provides reducing power in many enzymatic reactions that are crucial for macromolecular biosynthesis [73].NADH is also an antioxidant and forms part of the defense against reactive oxygen species (ROS) that are produced during rapid proliferation [74]. High levels of ROS can cause damage to macromolecules, which can induce senescence and apoptosis [75].

In our study, platelet degranulation plays an essential role in the biological process category of gene ontology analysis. Extensive experimental evidence shows that 


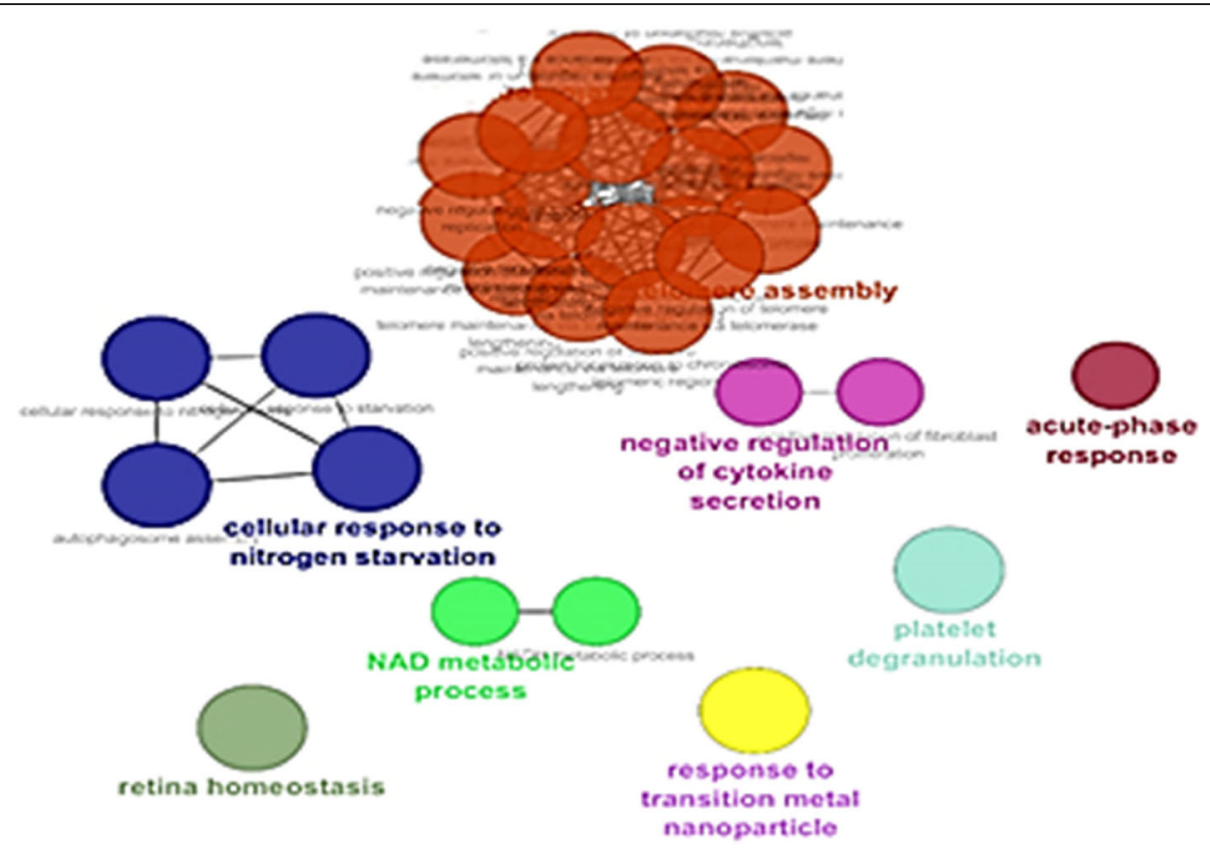

Fig. 3 Biological process category of gene ontology analysis based on Clupedia/CluGO with identified proteins

platelets support tumor metastasis [76]. Platelet activation and coagulation system play an important role in cancer progression [77].

According to the biological process category of gene ontology analysis, retina homeostasis may play a critical role in low-grade serous ovarian cancer. A breakthrough in Kessler's study, understanding of the molecular biology of ovarian cancer may depend on gaining a deeper insight into retina homeostasis [78].

The biological processes are relevant by acute phase in tumor cells. The host response comprises a cascade of inflammatory signals that can be triggered by small inciting events, e.g., localized infection or a small tumor, and that leads to up- and down-regulation of a group of

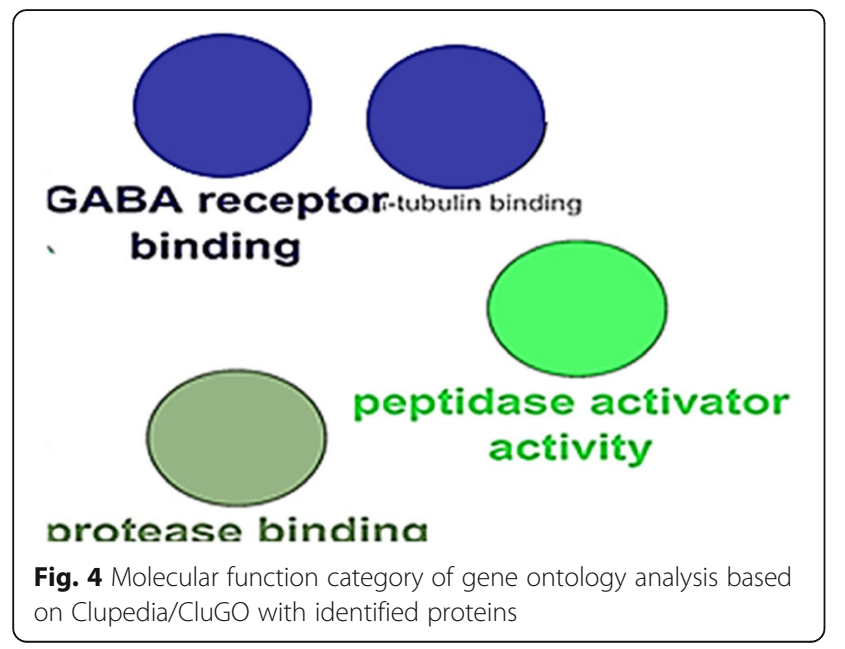

circulating proteins often called acute phase reactants [79]. Acute phase response in ovarian cancer earliest stages [80].

Iron is the most common metal in the human body. Epidemiological studies show that asbestos transition metal that catalyzes free radical generation is more carcinogenic [81].

Molecular function analysis showed that GABA receptor binding, peptidase activator, Protease binding are the involved function in low-grade serous ovarian cancer.

GABA receptor binding in human cancers may play a critical role in low-grade serous ovarian cancer.

MiR-224 is deregulated expression in various cancers, including cervical cancer, ovarian cancer, and lung adenocarcinoma [82].MiR-224 is located in the gammaaminobutyric acid (GABA), a receptor epsilon gene (GABRE) [83], and its expression is directly activated by E2F1through transactivation of the GABRE gene [84].P53 and p65 bind with miR-224 host gene and inactivate the GABAA receptor $\varepsilon$ subunit promoter in ovarian cancer [85].

Proteolytic enzymes such as peptidase have been implicated in the progression of various human malignancies, including ovarian cancer. Possibility for the role of peptidase activity in the tumor may initiate or terminate some biological events [86]. Thus, the importance of enhanced peptidase activity for malignant growth could originate from its possible regulatory role in RAS [87]. Simaga and et al. in their study on assessing the activity of peptidase activator in ovarian tissue, found that progression from benign into malignant transformation in 


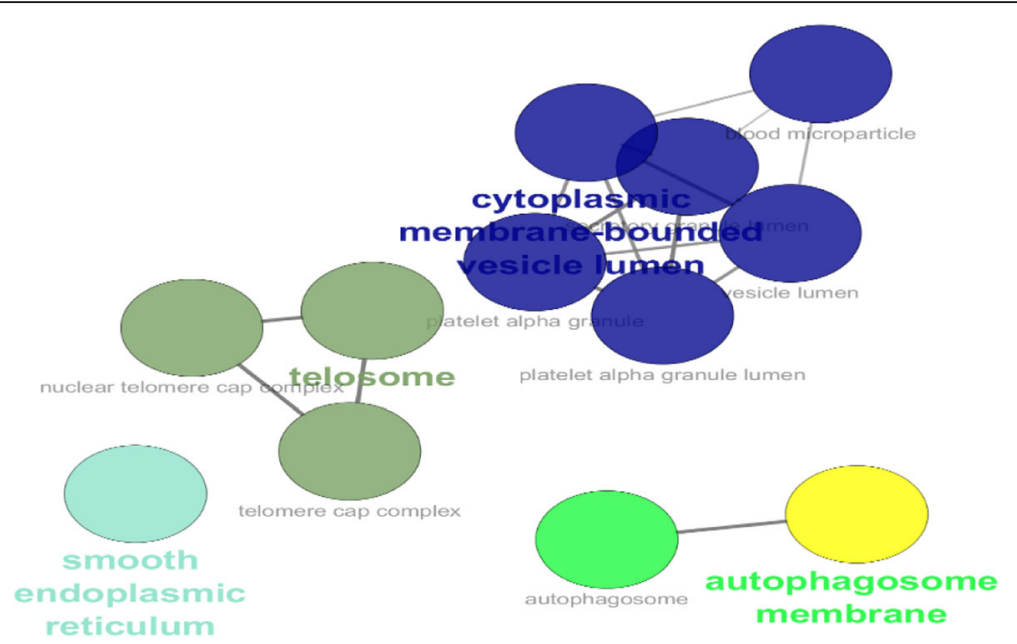

Fig. 5 Cellular components category of gene ontology analysis based on Clupedia/CluGO with identified proteins

ovarian tissue is accompanied with up-regulation of this proteolytic enzyme Such as our study [88].

Protease binding was one of the functions found inprotein-protein interaction in low-grade serous ovarian cancer in our study. Proteolytic activity is also very important at multiple stages during the intraperitoneal metastases of spheroids, especially for their initial detachment from the surface of the ovary. Since a number of published experimental studies emphasize the importance of adhesion molecules and proteases in spheroid formation, maintenance, and the subsequent adhesion of cancer cells at the secondary site, targeting their action makes biological sense [89].

Cell components of proteins are identified and based on Cytoplasmic membrane-bounded vesicle lumen, telesome, autophagosome membrane, and smooth endoplasmic reticulum.

In one study by Gilks on coexistence of intracytoplasmic lumens and membrane-bound vesicles in an invasive carcinoma, the formation of cytoplasmic lumen and membrane-bound vesicles involves two entirely separate processes that can coexist within a neoplastic cell [90].

The cell components are relevant by telesome in neoplasm cells. It is noteworthy that interference with telomeres, through direct targeting of telomeric DNA or proteins involved in the complex telomosum, can negatively affect the potential of not only tumors that express the activity of telomerase, but also those whose telomeres are transmitted through maintain unknown mechanisms [91].

In Zhen's study on roles of the autophagosome membrane in ovarian cancer cells, They identified that autophagic cell death was reduced when cultured human ovarian cancer cells in which gene had been reexpressed were treated with growth factors, angiogenic factors, and matrix proteins found in xenografts [92]
Autophagosomes then fuse with lysosomes, releasing their contents for hydrolysis [93].

The endoplasmic reticulum (ER) is responsible for the regulation of intracellular calcium $(\mathrm{Ca} 2+)$ and the synthesis of cell surface or secretory proteins [94]. However, ER stress will induce apoptotic death if homeostatic mechanisms are insufficient to protect or repair the cell [95].

Conclusion: Biomarker discovery and molecular investigation are potent tools in the diagnosis and treatment of this disease. Protein-protein interaction network analysis increases the understanding of molecular events [96]. Here, six proteins were introduced as hubbottleneck protein. It can be concluded that regulation of gene expression, including TAGLN, KRT14, ACTB, APOA1, PRDX2 and HP proteins can play a crucial role in the pathology of Low grade serous ovarian cancer.

\section{Acknowledgments}

This project has been executed by Shahid Beheshti University of Medical Sciences. This study is extracted from a Ph.D. thesis. Moreover, we would like to thank Shahid Beheshti Proteomics Research Center and Ms. Fatemeh Javadi for proofreading the manuscript.

Authors' contributions

All authors had an equal role in the design, work, statistical analysis, and manuscript writing. All authors read and approved the final manuscript.

\section{Funding}

Shahid Beheshti Medical Science

Availability of data and materials Not applicable.

\section{Ethics approval and consent to participate}

Ethics committee of Shahid Beheshti University of Medical Sciences approved the PhD thesis (Confirmation code: IR.SBMU.RETECH.REC.1396.709).

Consent for publication

Not applicable.

Competing interests

The authors declare that they have no competing interests. 


\section{Author details}

'Proteomics Research Center, Shahid Beheshti University of Medical Sciences, Tehran, Iran. ${ }^{2}$ Proteomics Research Center, School of Advanced Technologies in Medicine, Shahid Beheshti University of Medical Sciences, Tehran, Iran. ${ }^{3}$ Department of Gynecology, Guilan University of Medical Sciences, Rasht, Iran. ${ }^{4}$ Department of Genetics, Reproductive Biomedicine Research Center, Royan Institute, ACECR, Tehran, Iran.

\section{Received: 19 November 2018 Accepted: 28 June 2019}

\section{Published online: 17 July 2019}

\section{References}

1. Siegel R, Naishadham D, Jemal A. Cancer statistics, 2013. CA Cancer J Clin. 2013;63(1):11-30

2. Arab M, Khayamzadeh M, Hashemi M, Hosseini M, Tabatabaeefar M, Anbiaee R, Anvari N, Ebrahimi M, Akbari ME. Crude and age-specific incidence rate patterns for histopathologic subtypes of ovarian cancer in Iran. Arch Iran Med. 2010;13(3):203-8.

3. Cho KR, Shih I. Ovarian cancer. Annu Rev Pathol. 2009;4:287-313.

4. Bast RC Jr, Hennessy B, Mills GB. The biology of ovarian cancer: new opportunities for translation. Nat Rev Cancer. 2009;9:415-28.

5. TCGA. Integrated genomic analyses of ovarian carcinoma. Nature. 2011; 474(7353):609-15.

6. McGuire WP. Maintenance therapy for ovarian cancer: of Helsinki and Hippocrates. J Clin Oncol. 2009;27:4633-4.

7. Coleman MP, Forman D, Bryant H, Butler J, Rachet B, Maringe C. Cancer survival in Australia, Canada, Denmark, Norway, Sweden, and the UK, 19952007 (the International Cancer Benchmarking Partnership): an analysis of population-based cancer registry data. Lancet. 2011;377:127-38.

8. Seidman JD, Zhao P, Yemelyanova A. "Primary peritoneal" high-grade serous carcinoma is very likely metastatic from serous tubalintra epithelial carcinoma: assessing the new paradigm of ovarian and pelvic serous carcinogenesis and its plication's for screening for ovarian cancer. Gynecol Oncol. 2011;120(3):470-3.

9. Lee $Y$, Miron A, Drapkin R, Nucci MR, Medeiros F, Saleemuddin A, et al. A candidate precursor to serous carcinoma that originates in the distal fallopian tube. J Pathol. 2007;211:26-35.

10. Felix L, Natasha M, Eleftherios P, Diamandisa, Vathany K. Advances in mass spectrometry-based technologies to direct personalized medicine in ovarian cancer. Transl Proteomic. 2013;56:76-84.

11. Jones CM, Monge ME, Kim J, Matzuk MM, Fernández FM. Metabolomic serum profiling detects early-stage high-grade serous ovarian cancer in a mouse model. J Proteome Res. 2015 Feb 6;14(2):917-27.

12. Bhatla N, Denny L. FIGO cancer report 2018. Int J Gynaecol Obstet. 2018; 143:2-3 ISSN: 1879-3479

13. Markman M, Federico M, Liu PY, Hannigan E, Alberts D. Significance of early changes in the serum CA-125 antigen level on overall survival in advanced ovarian cancer. Gynecol Oncol. 2006;103(1):195-8.

14. Kolwijck E, Span PN, Thomas CM, Bulten J, Sweep FC, Massuger LF. Prognostic value of CA 125 in ovarian cyst fluid of patients with epithelial ovarian cancer. Oncol Rep. 2010;23(2):579-84.

15. Kolwijck E, Zusterzeel PL, Roelofs HM, Hendriks JC, Peters WH, Massuger LF GSTP1-1 in ovarian cyst fluid and disease outcome of patients withovarian cancer. Cancer Epidemiol Biomarkers Prev. 2009;18(8):2176-81.

16. Moore RG, Brown AK, Miller MC, Skates S, Allard WJ, Verch T, Steinhoff M, Messerlian G, DiSilvestro P, Granai CO. The use of multiple novel tumor biomarkers for the detection of ovarian carcinoma in patients with a pelvic mass. Gynecol Oncol. 2008;108(2):402-8.

17. Wright PC, Noirel J, Ow SY, Fazeli A. A review of current proteomics technologies with a survey on their widespread use in reproductive biology investigations. Theriogenology. 2012;77(738-65):e52.

18. Hogdall C, Fung ET, Christensen IJ, Nedergaard L, Engelholm SA, Petri AL, et al. A novel proteomic biomarker panel as a diagnostic tool for patients with ovarian cancer. Gynecol Oncol. 2011:123:308-13.

19. Petri AL, Simonsen AH, Yip TT, Hogdall E, Fung ET, Lundvall L, Hogdal C. Three new potential ovarian cancer biomarkers detected in human urine with equalizer bead technology. Acta Obstet Gynecol Scand. 2009:88(1):18-26.

20. Toyama A, Suzuki A, Shimada T, Aoki C, Aoki Y, Umino Y, Nakamura Y, Aok $D$, Sato TA. Proteomic characterization of ovarian ancers identifying annexin-A4, phosphoserine aminotransferase, cellular retinoic acidbinding protein 2, and serpin B5 as histology-specific biomarkers. Cancer Sci. 2012; 103(4):747-55.

21. Kristjansdottir B, Kristina L, Partheen K, Carlsohn E, Sundfeldt K. Potential tumor biomarkers identified in ovarian cyst fluid by quantitative proteomic analysis, iTRAQ. Clin Proteomics. 2013;10:4.

22. Kim DC, Wang X, Yang CR, Gao JX. A frameworkfor personalized medicine: prediction of drug sensitivity in cancer by proteomic profiling. Proteome Sci. 2012;10:1-13.

23. Morvaj H, Zali A, Zali H, Rezaee TM, Rastegar F. Biomarkers of basal cell carcinoma using proteomic analysis. J Pajohandeh. 2009;3(69):137-41.

24. Zhou G, Li H, DeCamp D, Chen S, Shu H, Gong Y. 2D differential in-gel electrophoresis for the identification of esophageal scans cell cancerspecific protein markers. Mol Cell Proteomics. 2002;1(2):117-24.

25. Shannon P, Markiel A, Ozier O, Baliga NS, Wang JT, Ramage D, Amin N, Schwikowski B, Ideker T. Cytoscape: a software environment for integrated models ofbiomolecular interaction networks. Genome Res. 2003:13:2498-504

26. Safari-Alighiarloo N, Rezaei-Tavirani M, Taghizadeh M, Tabatabaei S, Namaki S. Network-based analysis of differentially expressed genes in cerebrospinal fluid (CSF) and blood reveals new candidate genes for multiple sclerosis. PeerJ. 2016:4:2775

27. Vergara D, Tinelli A, Martignago R, Malvasi A, Leo G. Biomolecular pathogenesis of borderline ovarian tumors: focusing target discovery through proteogenomics. Curr Cancer Drug Targets. 2010;10:107-16.

28. Kim SW, Kim S, Nam EJ, Jeong YW, Lee SH, Paek JH, et al. Comparative proteomic analysis of advanced serous epithelial ovarian carcinoma: possible predictors of chemo resistant disease. OMICS. 2011;15:281-92.

29. Pan S, Cheng L, White JT, Lu W, Utleg AG, Yan X, et al. Quantitative proteomics analysis integrated with microarray data reveals that extracellular matrix proteins, catenins, and p53 binding protein 1 are important for chemotherapy response in ovarian cancers. OMICS. 2009;13: 345-54.

30. Jinawath $\mathrm{N}$, Vasoontara C, Jinawath A, Fang X, Zhao K, Yap KL, et al. Oncoproteomic analysis reveals co-upregulation of RELA and STAT5 in carboplatin resistant ovarian carcinoma. PLoS One. 2010;5:e11198.

31. El Ayed M, Bonnel D, Lonquespée R, Castellier C, Franck J, Vergara D. MALDI imaging mass spectrometry in ovarian cancer for tracking, identifying, and validating biomarkers. Med Sci Monit. 2010;16(8):BR233-45.

32. Zhou $H$, Fang $Y$, Weinberger $P$, Ding $P$, Cowell $K$, Hudson F, Ren M, Jeffrey $R$. Transgelin increases metastatic potential of colorectal cancer cells in vivo and alters expression of genes involved in cell motility. BMC Cancer. 2016; 16:48-55.

33. Keshamouni VG, Michailidis G, Grasso CS, et al. Differential protein expression profiling by iTRAQ-2DLC-MS/MS of lung cancer cells undergoing epithelial-mesenchymal transition eveals a migratory/invasive phenotype. J Proteome Res. 2006:5:1143-54

34. Lin Y, Buckhaults PJ, Lee JR, Xiong H, Farrell C, Podolsky RH, et al. Association of the actin-binding protein transgelin with lymph node metastasis in human colorectal cancer. Neoplasia. 2009:11:864-73.

35. Giefing M, Zemke N, Brauze D, Kostrzewska-Poczekaj M, Luczak M, Szaumkessel $M$, et al. High resolution ArrayCGH and expression profiling identifies PTPRD and PCDH17/PCH68 as tumor suppressor gene candidates in laryngeal squamous cell carcinoma. Genes Chromosomes Cancer. 2011 50:154-66.

36. Ogawa A, Sakatsume M, Wang X, Sakamaki Y, Tsubata Y, Alchi B, et al. SM22alpha: the novel phenotype marker of injured glomerular epithelia cells in anti-glomerular basement membrane nephritis. Nephron Exp Nephrol. 2007;106:e77-87.

37. Yu H, Konigshoff M, Jayachandran A, Handley D, Seeger W, Kaminski N, et al. Transgelin is a direct target of TGF-beta/Smad3-ependent epithelial cell migration in lung fibrosis. FASEB J. 2008;22:1778-89.

38. Dvorakova M, Nenutil R, Bouchal P. Transgelins, cytoskeletal proteins implicated in different aspects of cancer development. Expert Rev Proteomics. 2014:11:149-65.

39. Pantel $\mathrm{K}$, Brakenhoff $\mathrm{RH}$, Brandt $\mathrm{B}$. Detection, clinical relevance and specific biological properties of disseminating tumor cells. Nat Rev Cancer. 2008;8: 329-40.

40. Riethdorf S, Fritsche H, Muller V, Rau T, Schindlbeck C, Rack B, et al. Detection of irculating tumor cells in peripheral blood of patients with metastatic breast cancer: a validation study of the cell search system. Clin Cancer Res. 2007;13:920-8. 
41. Kitagawa K, et al. Epithelial mesenchymal transformation of a newly established cell line from ovarian adenocarcinoma by transforming growth factor-beta1. Int J Cancer. 1996;66:91-7.

42. Vergara D, Merlot B, Lucot JP, et al. Epithelial-mesenchymal transition in ovarian cancer. Cancer Lett. 2010;291:59-66.

43. Joosse S, Hannemann J, Spötter J, Bauche A, Andreas A. Changes in keratin expression during metastatic progression of breast cancer: impact on the detection of circulating tumor cells. Clin Cancer Res. 2012;4:15-8.

44. Toyama A, Suzuki A, Shimada T, Aoki C, Aoki Y, Umino Y. Proteomic characterization of ovarian cancers identifying annexin-A4, phosphoserine aminotransferase, cellular retinoic acid-binding protein 2, and serpin B5 as histology-specific biomarkers. Cancer Sci. 2012;103:747-55.

45. Zhou Y, Xiaofang $Y$, Stoffer J, Bonafe N. The multifunctional protein glyceraldehyde-3-phosphatedehydrogenase is both regulated and controls colony-stimulating factor-1 messenger RNA stability in ovarian cancer. Mol Cancer Res. 2008;7:6-8

46. Epner DE, Coffey DS. There are multiple forms of glyceraldehyde-3dehydrogenase in prostate cancer cells and normal prostate tissue. Prostate. 1996;28:372-8.

47. El Ayed M, Bonnel D,Longuespee R,Castelier C, Franck J. MALDI imaging mass spectrometry in ovarian cancer for tracking, identifying, and validating biomarkers. Med Sci Monit. 2010;16(8):233-45.

48. Cortesi L, Rossi E, Della Casa L, Barchetti A, Nicoli A. Protein expression patterns associated with advanced stage ovarian cancer. Electrophoresis. 2011;32:1-12.

49. Abdel-Azeez HA, Labib HA, Sharaf SM, Refai AN. HE4 and mesothelin: novel biomarkers of ovarian carcinoma in patients with pelvic masses. Asian Pac J Cancer Prev. 2010;11(1):111-6.

50. Nosov V, Su F, Amneus M, et al. Validation of serum biomarkers for detection of early-stage ovarian cancer. Am J Obstet Gynecol. 2009;200:639.e1-5.

51. Kim KD, Lim HY, Lee HG, et al. Apolipoprotein A-I induces IL-10 and PGE2 production in human monocytes and inhibits dendritic cell differentiation and maturation. Biochem Biophys Res Commun. 2005;338:1126-36.

52. Saga $Y$, Ohwada M, Suzuki $M$, et al. Glutathione peroxidase 3 is a candidate mechanism of anticancer drug resistance of ovarian clear cell adenocarcinoma. Oncol Rep. 2008;20:1299-303.

53. Chung YM, Yoo YD, Park JK, Kim YT, Kim HJ. Increased expression of peroxiredoxin II confers resistance to cisplatin. Anticancer Res. 2001:21:1129-33.

54. Ahmed N, Barker G, Oliva K, Hoffmann P, Riley C, Reeve S. Proteomic-based identification of haptoglobin-1 precursor as a novel circulating biomarker of ovarian cancer. Br J Cancer. 2004;91:129-40.

55. Wassell J. Haptoglobin: function and polymorphism. Clin Lab. 2000;46: 547-52.

56. Misumi $Y$, Tanaka $Y$, Ikehara $Y$. Biosynthesis, intracellular processing and secretion of haptoglobin in cultured rat hepatocytes. Biochem Biophys Res. 1983;114:729-36.

57. Mueller WK, Handschumacher R, Wade ME. Serum haptoglobin in patients with ovarian malignancies. Obstet Gynecol. 1971;38:427-35.

58. Biton A, Bernard-Pierrot I, Lou Y, et al. Independent component analysis uncovers the landscape of the bladder tumor transcriptome and reveals insights into luminal and basal subtypes. Cell Rep. 2014;9:1235-45.

59. Nofech-Mozes S, Khalifa MA, Ismiil N, Saad RS, Hanna WM, Covens A, Ghorab Z. Immunophenotyping of serous carcinoma of the female genital tract. Mod Pathol. 2008;21:1147-55.

60. Ricciardelli C, Lokman N, Pyragius C. Keratin 5 overexpression is associated with serous ovarian cancer recurrence and chemotherapy resistance. Oncotarget. 2017:8(11):17819-32.

61. Capo-chichi C, Cai KQ, Xiang X. Overexpression and cytoplasmic localization of caspase- 6 is associated with lamin A degradation in set of ovarian cancers. Biomark Res. 2018;6:30-5.

62. Kozak KR, Su F, Whitelegge JP, Faull K, Reddy S, Farias-Eisner R. Characterization of serum biomarkers for detection of early stage ovarian cancer. Proteomics. 2005;5:4589-96.

63. Braicu E, Darb-Esfahani S, Schmitt W. High-grade ovarian serous carcinoma patients exhibit profound alterations in lipid metabolism. Oncotarget. 2017; 8(61):102912-20.

64. Li S, Hu X, Ye M, Zhu X. The prognostic values of the peroxiredoxins family in ovarian cancer. Biosci Rep. 2018;38:BSR20180667.

65. Mahyuddin AP, Liu L, Zhao C, Kothandaraman N. Diagnostic accuracy of haptoglobin within ovarian cyst fluid as a potential point-of-care test for epithelial ovarian cancer: an observational study. BJOG. 2018;125:421-43.
66. Tipney $H$, Hunter L. An introduction to effective use of enrichment analysis software. Hum Genomics. 2010:4:202-6.

67. Binns D, Dimmer E, Huntley R, Barrell D, O'Donovan C, Apweiler R. QuickGO: a web-based tool for gene ontology searching. Bioinformatics. 2009;25: 3045-6.

68. Baig S, Seevasant I, Mohamad J, Mukheem A, Huri A. Potential of apoptotic pathway-targeted cancer therapeutic research: where do we stand? Cell Death Dis. 2016;7:e2058.

69. Mao HL, Liu PS, Zheng JF, Zhang PH, Zhou LG, Xin G, et al. Transfection of Smac/DIABLO sensitizes drug-resistant tumor cells to TRAIL or paclitaxelinduced apoptosis in vitro. Pharmacol Res. 2007;56:483-92.

70. Gary A, Ji-Fan H, Thanh H, Haritha O. Regulation of telomerase by alternate splicing of human telomerase reverse transcriptase (hTERT) in normal and neoplastic ovary, endometrium and myometrium. Int J Cancer. 2000:85:330-5.

71. Miyahara Y, Odunsi K, Chen W. Generation and regulation of human CD4_ LL-17-producing T cells in ovarian cancer. PNAS. 2008;105(40):15505-10.

72. Czerniecki B, Koski G, Koldovsky U, Shuwen X. Targeting HER-2/neu in early breast cancer development using dendritic cells with staged interleukin-12 burst secretion. Cancer Res. 2007;67:4-10.

73. Huarte E, Cubillos-Ruiz J, Nesbeth Y, Scarlett U, Martinez D, Buckanovich R, Benencia, et al. Depletion of dendritic cells delays ovarian cancer progression by boosting antitumor immunity. Cancer Res. 2008;68:7684-91.

74. Kurman RJ, Shih I. Molecular pathogenesis and extra ovarian origin of epithelial ovarian cancer-shifting the paradigm. Hum Pathol. 2011;42: 918-31.

75. Godwin A, Meister $\mathrm{P}, \mathrm{O}^{\prime}$ Dwyer $\mathrm{P}$, Huang CS, Hamilton TC. High resistance to cisplatinin human ovarian cancer cell lines is associated with marked increase of glutathione synthesis. Proc Natl Acad Sci U S A. 1992;89:3070-4.

76. Gay LJ, Brunhilde. Contribution of platelets to tumour metastasis. Cancer. 2011;11:123-35

77. Zaslavsky $\mathrm{A}$, et al. Platelet-derived thrombospondin-1 is a critical negative regulator and potential biomarkerof angiogenesis. Blood. 2010;115:4605-13.

78. Kessler M, Fotopoulou C, Meyer T. The molecular fingerprint of high grade serous ovarian cancer reflects its fallopian tube origin. Int J Mol Sci. 2013;14: 6571-96.

79. Gabay C, Kushner I. Acute-phase proteins and other systemic responses to inflammation. N Engl J Med. 1999:340:448-54.

80. Tognon G, Carnazza M, Ragnoli M, Calza S, Ferrari F, Gambino A, Zizioli V, Notaro S, Sostegni B, Sartori E. Prognostic factors in early-stage ovarian cancer. Ecancermedicalscience. 2013;7:325

81. Toyokuni S. Role of iron in carcinogenesis: cancer as a ferrotoxic disease. Cancer Sci. 2009;100(1):9-16

82. Liang M, Yao GD, Yin MM, Lu M, Tian H, Liu L, et al. Transcriptional cooperation between p53 and NF-kB p65 regulates microRNA-224 transcription in mouse ovarian granulosa cells. Mol Cell Endocrinol. 2013; 370(1-2):119-29.

83. Chen W, Fan X-m, Mao L. MicroRNA-224: as a potential target for miRbased therapy of cancer. Tumor Biol. 2015. https://doi.org/10.1007/s132 77-015-3883-3.

84. Knoll S, Fürst K, Kowtharapu B, Schmitz U, Marquardt S, Wolkenhauer O, et al. E2F1 induces miR-224/452 expression to drive EMT through TXNIP downregulation. EMBO Rep. 2014;15(12):1315-29.

85. Song GQ, Song GR, Ni HY, Gu L, Liu H, Chen B, et al. Deregulated expression of miR-224 and its target gene: CD59 predicts outcome of diffuse large B-cell lymphoma patients treated with R-CHOP. Curr Cancer Drug Targets. 2014;14(7):659-7.

86. Kerins DM, Hao Q, Vaughan DE. Angiotensin induction of PAl-1expression in endothelial cells is mediated by the hexapeptide angiotensin IV. J Clin Invest. 1995;96:2515-20

87. Lengyel E. Ovarian cancer development and metastasis. AJP. 2010;177(3): 1053-64.

88. Simaga S, Babic D, Osmak B. Tumor cytosol dipeptidyl peptidase III activity is increased with histological aggressiveness of ovarian primary carcinomas. Gynecol Oncol. 2003;5:194-200.

89. Stadler M, Scherzer M, Walter S, Holzner S, Pudelko K. Exclusion from spheroid formation identifies loss of essential cell-cell adhesion molecules in colon cancer cells. Sci Rep. 2018:8:1151-62.

90. Gilks B, Hvassoli F. Coexistence of intracytoplasmic lumensand membranebound vesicles in an invasive carcinoma arising in a cystosarcomaphyllodes. Ultrastruct Pathol. 1988;12:631-42. 
91. Folini M, Gandellini P, Zaffaroni N. Targeting the telosome: therapeutic implications. Biochim Biophys Acta. 2009;10:309-16.

92. Lu Z, Robert Z, et al. The tumor suppressor gene ARHI regulates autophagy and tumor dormancy in human ovarian cancer cells. J Clin Invest. 2008; 118(12):3917-29.

93. Jin $\mathrm{S}$, White $\mathrm{E}$. Role of autophagy in cancer: management of metabolic stress. Autophagy. 2007;3:28-31.

94. Penny E, Corazzari M, Jane L. Increasing melanoma cell death using inhibitors of protein disulfide isomerases to abrogate survival responses to endoplasmic reticulum stress. Cancer Res. 2008;68(13):5363-9.

95. Szegezdi E, Logue SE, Gorman AM, Samali A. Mediators of endoplasmic reticulum stress-induced apoptosis. EMBO Rep. 2006;7:880-5.

96. Safaei A, Tavirani MR, Oskouei AA, Azodi MZ. Protein-protein interaction network analysis of cirrhosis liver disease. Gastroenterol Hepatol Bed Bench. 2016;9(2):114-23.

\section{Publisher's Note}

Springer Nature remains neutral with regard to jurisdictional claims in published maps and institutional affiliations.

Ready to submit your research? Choose BMC and benefit from:

- fast, convenient online submission

- thorough peer review by experienced researchers in your field

- rapid publication on acceptance

- support for research data, including large and complex data types

- gold Open Access which fosters wider collaboration and increased citations

- maximum visibility for your research: over $100 \mathrm{M}$ website views per year

At $\mathrm{BMC}$, research is always in progress.

Learn more biomedcentral.com/submissions 